\title{
A CLASS OF PURE SUBGROUPS OF COMPLETELY DECOMPOSABLE ABELIAN GROUPS
}

\author{
DAVID M. ARNOLD
}

\begin{abstract}
Direct sum decompositions of the class of pure subgroups of finite rank completely decomposable torsion free abelian groups with typesets of cardinality at most 4 are considered. In certain cases, the indecomposable groups are classified, resulting in new proofs of several theorems by T. B. Cruddis.
\end{abstract}

The class $\mathscr{R}$ of pure subgroups of completely decomposable torsion free abelian groups of finite rank is considered by Butler [2]. Many of the examples of pathological direct sum decompositions of finite rank torsion free abelian groups occur in the class $\mathscr{R}$. On the other hand, homogeneous $\mathscr{R}$-groups are completely decomposable and if $A$ is an $\mathscr{R}$-group, then typeset $(A)$ is finite and closed under intersection of types.

This note considers $\mathscr{R}$-groups with typesets of cardinality at most 4 . Motivation is provided by the curious results of Cruddis [3]. An easier and more illuminating proof of these results is a consequence of the following theorems.

Let $\Lambda=\left\{\tau_{0}, \tau_{1}, \tau_{2}, \tau_{3}\right\}$ be a set of types with $\tau_{0}=\tau_{1} \cap \tau_{2} \cap \tau_{3}$; let $\mathscr{R}_{\Lambda}$ be the class of $\mathscr{R}$-groups with typeset $\subseteq \Lambda$; let $\Sigma_{\Lambda}$ be the sum of types in $\Lambda$; and let $\sum_{A}$ be the sum of types in typeset $(A)$. Define $\tau_{\infty}$ to be the type represented by $(\infty)$ and, for $p$ a prime, let $\tau_{p}$ be the type represented by $\left(n_{q}\right)$ where $n_{p}=0$ and $n_{q}=\infty$ if $p \neq q$.

THEOREM 1. Suppose that $A$ is an $\mathscr{R}_{\Lambda}$-group and that typeset $(A)$ is a proper subset of $\Lambda$.

(a) If $A$ is indecomposable, then rank $A \leqq 2$.

(b) If $\sum_{A}=\tau_{\infty}$, then $A$ is completely decomposable.

(c) If $\sum_{A}=\tau_{p}$, for some prime $p$, then $A$ has a unique decomposition (up to isomorphism) into indecomposable summands.

THeOREM 2. Suppose that $A$ is an $\mathscr{R}_{\Lambda}$-group with typeset $(A)=\Lambda$ and that $\sum_{\Lambda}=\tau_{\infty}$.

(a) If $A$ is indecomposable, then rank $A \leqq 3$.

Received by the editors February 7, 1973.

AMS (MOS) subject classifications (1970). Primary $20 \mathrm{~K} 15$.

Key words and phrases. Torsion free abelian groups of finite rank, completely decomposable groups.

(c) American Mathematical Society 1973 
(b) There is a completely decomposable $\mathscr{R}_{\Lambda}$-group $H$ such that $A \oplus H=G_{1} \oplus \cdots \oplus G_{k}$ and $\operatorname{rank}\left(G_{i}\right) \leqq 2$ for $1 \leqq i \leqq k$.

Corollary 1.2 includes an explicit characterization of all indecomposable $\mathscr{R}_{\Lambda}$-groups, where $\sum_{\Lambda}=\tau_{\infty}$.

Let $\pi=\{p, q, r\}$ be a set of distinct primes, let $\mathscr{C}_{3}$ be the class of $\mathscr{R}$ groups divisible by all primes not in $\pi$, and let $\Lambda=\left\{\tau_{0}, \tau_{1}, \tau_{2}, \tau_{3}\right\}$ where $\tau_{0}=\tau_{p} \cap \tau_{q} \cap \tau_{r}, \tau_{1}=\tau_{p} \cap \tau_{q}, \tau_{2}=\tau_{p} \cap \tau_{r}$ and $\tau_{3}=\tau_{q} \cap \tau_{r}$.

Corollary 3 (CRUdDis). Let $A$ be a $\mathscr{C}_{3}$-group.

(a) $A=H \oplus G$ where $H$ is completely decomposable and $G$ is an $\mathscr{R}_{\Lambda^{-}}$-group.

(b) Any two decompositions of $A$ into indecomposable summands with rank $\leqq 2$ are equivalent.

(c) $K_{0}\left(\mathscr{C}_{3}\right)$ is a free abelian group with $S=\{[A] \mid A$ is indecomposable and rank $A \leqq 2\}$ as a basis $\left(K_{0}\left(\mathscr{C}_{3}\right)\right.$ is the Grothendieck group of $\mathscr{C}_{3}$, modulo direct sums, and $[A]$ is the element of $K_{0}\left(\mathscr{C}_{3}\right)$ determined by $\left.A\right)$.

0 . Preliminaries and notation. The basic reference for the elementary notions of torsion free abelian groups is Fuchs [4].

A height sequence is a sequence $\left(n_{p}\right)$ of extended integers indexed by the set of primes of $Z$, such that $0 \leqq n_{p} \leqq \infty$ for each prime $p$. Two height sequences $\left(n_{p}\right)$ and $\left(m_{p}\right)$ are equivalent if $n_{p}=m_{p}$ for all but a finite number of primes $p$ with $n_{p}<\infty$ and $m_{p}<\infty$. A type is an equivalence class of height sequences. The natural ordering of height sequences induces an ordering on the set of all types (we write $\tau_{1} \cap \tau_{2}$ for $\min \left\{\tau_{1}, \tau_{2}\right\}$ ) and addition of height sequences (with the convention that $\infty+k=\infty$ ) induces addition of types.

Assume that $A$ is a torsion free abelian group, that $0 \neq a \in A$ and that $p$ is a prime. Then $h_{p}(a)$ is the largest integer $n$ with $a \in p^{n} A \backslash p^{n+1} A$ and $\infty$ if no such $n$ exists. Define $h_{A}(a)$ to be the height sequence $\left(h_{p}(a)\right)$; the type $T_{A}(a)$ of $a$ in $A$ is the equivalent class of $h_{p}(a)$. Note that

$$
T_{A}\left(a_{1}+\cdots+a_{n}\right) \geqq T_{A}\left(a_{1}\right) \cap \cdots \cap T_{A}\left(a_{n}\right)
$$

and if $A=A_{1} \oplus \cdots \oplus A_{n}$ with $a_{i} \in A_{i}$, then

$$
T_{A}\left(a_{1}+\cdots+a_{n}\right)=T_{A}\left(a_{1}\right) \cap \cdots \cap T_{A}\left(a_{n}\right) .
$$

Define typeset $(A)$ to be $\left\{T_{A}(a) \mid 0 \neq a \in A\right\} ; A$ is homogeneous if typeset $(A)$ is a singleton. If rank $A=1$, then $A$ is homogeneous. Call $A$ completely decomposable if $A$ is the direct sum of rank 1 groups. Pure subgroups of finite rank homogeneous completely decomposable groups are summands.

If $\tau$ is a type, then $A(\tau)=\left\{x \in A \mid T_{A}(x) \geqq \tau\right\}$ is a pure fully invariant subgroup of $A$. If $A=B \oplus C$, then $A(\tau)=B(\tau) \oplus C(\tau)$ and $A / A(\tau)$ is isomorphic to $B / B(\tau) \oplus C / C(\tau)$. Two abelian groups $A$ and $B$ are quasiisomorphic if there are isomorphic subgroups $A^{\prime}$ and $B^{\prime}$ of $A$ and $B$, 
respectively, such that $A / A^{\prime}$ and $B / B^{\prime}$ are bounded. The group $A$ is strongly indecomposable if $A$ quasi-isomorphic to $B \oplus C$ implies that $B=0$ or $C=0$.

\section{1. $\mathscr{R}_{\Lambda}$-groups.}

Lemma 1.1 (ButLer [2]). Let $A$ be an $\mathscr{R}$-group with typeset(A) where $\tau_{0}=\tau_{1} \cap \cdots \cap \tau_{n}$.

(a) If typeset $(A)$ is well ordered, then $A$ is completely decomposable.

(b) If $A$ is indecomposable, then $A /\left(A\left(\tau_{1}\right)+\cdots+A\left(\tau_{n}\right)\right)$ is a finite group. Moreover, if $\tau_{0}=\tau_{i} \cap \tau_{j}$ for $1 \leqq i \neq j \leqq n$, then $A\left(\tau_{i}\right) \cap A\left(\tau_{j}\right)=0$ whenever $i \neq j$.

ProOF OF THEOREM 1. In view of Lemma 1.1(a), we may assume that typeset $(A)=\left\{\tau_{0}, \tau_{1}, \tau_{2}\right\}$ where $\tau_{0}=\tau_{1} \cap \tau_{2}, \tau_{0} \neq \tau_{1}$, and $\tau_{0} \neq \tau_{2}$. If $A$ is indecomposable, then $A /\left(A\left(\tau_{1}\right) \oplus A\left(\tau_{2}\right)\right)=C_{1} \oplus \cdots \oplus C_{k}$, where each $C_{i}$ is a cyclic group of order $m_{i}$ and $m_{i}$ divides $m_{i+1}$. Let $A=A\left(\tau_{1}\right)+A\left(\tau_{2}\right)+$ $Z x_{1}+\cdots+Z x_{k}$, where $x_{i}+A\left(\tau_{1}\right)+A\left(\tau_{2}\right)$ is a generator of $C_{i}$ and $m_{i} x_{i}=$ $a_{1 i}+a_{2 i}$ for some $a_{j i} \in A\left(\tau_{j}\right)(Z x$ is the subgroup of $A$ generated by $x)$.

(a) Assume $A$ is indecomposable with rank $>1$ and let $G_{i}$ be the pure subgroup of $A$ generated by $\left\{a_{1 i}, a_{2 i}\right\}$ for $1 \leqq i \leqq k$. Note that $a_{1 i}$ and $a_{2 i}$ are both nonzero since $A\left(\tau_{j}\right)$ is a pure subgroup of $A$ and $C_{i} \neq 0$. Thus $\operatorname{rank}\left(G_{i}\right)=2$.

Let $S=\left\{a_{11}, \cdots, a_{1 k}, a_{21}, \cdots, a_{2 k}\right\}$ and suppose that $l_{1} a_{11}+\cdots+$ $l_{k} a_{1 k}+n_{1} a_{21}+\cdots+n_{k} a_{2 k}=0$, for some $l_{i}, n_{i} \in Z$. Since $A\left(\tau_{1}\right) \cap A\left(\tau_{2}\right)=0$, $\sum l_{i} a_{1 i}=\sum n_{i} a_{2 i}=0$ and $\sum l_{i} m_{i} x_{i} \in A\left(\tau_{2}\right)$. Thus $\sum r_{i} x_{i} \in A\left(\tau_{2}\right)$, where $r_{i}=\left(l_{i} m_{i}\right) / d$ and $d$ is the greatest common divisor of $\left\{l_{1} m_{1}, \cdots, l_{k} m_{k}\right\}$. Consequently, $\sum\left(r_{i} x_{i}+A\left(\tau_{1}\right)+A\left(\tau_{2}\right)\right)=0 \in C_{1} \oplus \cdots \oplus C_{k}$ and $m_{1}$ divides all $r_{i}$, a contradiction unless $l_{1}=l_{2}=\cdots=l_{k}=0$. Similarly, $n_{i}=0$ for $1 \leqq i \leqq k$ and $S$ is a $Z$-independent subset of $A$.

One can easily verify that $G=G_{1} \oplus \cdots \oplus G_{k} \subseteq A$ and that $A\left(\tau_{j}\right)=$ $\left(G \cap A\left(\tau_{j}\right)\right) \oplus A_{j}^{\prime}$ for some $A_{j}^{\prime}$, where $j=1,2\left(A\left(\tau_{j}\right)\right.$ is a homogeneous completely decomposable group of type $\left.\tau_{j}\right)$. But $A\left(\tau_{1}\right) \oplus A\left(\tau_{2}\right) \subseteq G \oplus A_{1}^{\prime} \oplus A_{2}^{\prime} \subseteq A$ and $x_{1}, \cdots, x_{k} \in G$ so $A=G_{1} \oplus \cdots \oplus G_{k} \oplus A_{1}^{\prime} \oplus A_{2}^{\prime}$. Since $A$ is indecomposable, $A=G_{k}, k=1$, and $A /\left(A\left(\tau_{1}\right) \oplus A\left(\tau_{2}\right)\right)$ is cyclic.

(b) Assume that $A$ is indecomposable and that $\tau_{1}+\tau_{2}=\tau_{\infty}$. If $q a \in$ $A\left(\tau_{1}\right) \oplus A\left(\tau_{2}\right)$ for some prime $q$ and some $a \in A$, then $a \in A\left(\tau_{1}\right) \oplus A\left(\tau_{2}\right)$ since either $q A\left(\tau_{1}\right)=A\left(\tau_{1}\right)$ or $q A\left(\tau_{2}\right)=A\left(\tau_{2}\right)$. Consequently, $A=A\left(\tau_{1}\right) \oplus$ $A\left(\tau_{2}\right)$, a completely decomposable group.

(c) First assume that $A$ is indecomposable of rank 2, and that $\tau_{1}+\tau_{2}=\tau_{p}$ for some prime $p$. Then $N_{A}=A /\left(A\left(\tau_{1}\right) \oplus A\left(\tau_{2}\right)\right)$ is a cyclic group of order $p^{\alpha}$ for some $1 \leqq \alpha<\infty$ (see Theorem 1(a) and 1(b)). Let $B$ be another rank 2 indecomposable $\mathscr{R}_{\Lambda}$-group such that $\operatorname{typeset}(B)=\operatorname{typeset}(A)$ where $N_{B}$ is cyclic of order $p^{\beta}$. 
If $\alpha=\beta$ then there is a monomorphism $f: A \rightarrow B$ such that $f: A\left(\tau_{1}\right) \oplus$ $A\left(\tau_{2}\right) \rightarrow B\left(\tau_{1}\right) \oplus B\left(\tau_{2}\right)$ is an isomorphism. Let $A=A\left(\tau_{1}\right)+A\left(\tau_{2}\right)+Z x$, where $x+A\left(\tau_{1}\right)+A\left(\tau_{2}\right)$ is a generator of $N_{A}$, and let $i$ be the least positive integer with $p^{i} f(x) \in B\left(\tau_{1}\right) \oplus B\left(\tau_{2}\right)=f\left(A\left(\tau_{1}\right) \oplus A\left(\tau_{2}\right)\right)$. Since $f$ is monic, $i=\alpha$ and $f$ is an isomorphism. Thus $A \simeq B$ iff $\alpha=\beta$.

Let $A=A_{1} \oplus \cdots \oplus A_{m}=B_{1} \oplus \cdots \oplus B_{n}$ be two decompositions of $A$ into indecomposable summands. Observe that $N_{A}$ is the direct sum of a finite number of copies of cyclic $p$-groups and a finite number of rank 1 groups of type $\tau_{0}$. It now follows that $m=n$ and $A_{i} \simeq B_{\sigma(i)}$ for some permutation $\sigma$ of $\{1,2, \cdots, n\}$ (use the preceding remarks and the fact that $N_{A} \simeq N_{A_{1}} \oplus$ $\left.\cdots \oplus N_{A_{m}} \simeq N_{B_{1}} \oplus \cdots \oplus N_{B_{n}}\right)$.

Proof of Theorem 2(a). Assume that $A$ is indecomposable of rank $\geqq 2$, denote $A\left(\tau_{j}\right)$ by $A_{j}, j=1,2,3$, and write $A /\left(A_{1}+A_{2}+A_{3}\right)=$ $C_{1} \oplus \cdots \oplus C_{k}$, where $C_{i}$ is a cyclic group of order $m_{i}$ and $m_{i}$ divides $m_{i+1}$. Then $A=A_{1}+A_{2}+A_{3}+Z x_{1}+\cdots+Z x_{k}$, where $x_{i}+A_{1}+A_{2}+A_{3}$ is a generator of $C_{i}, m_{i} x_{i}=x_{1 i}+x_{2 i}+x_{3 i}$, and $x_{j i} \in A_{j}$.

Define $S_{j}=\left\{x_{j i} \neq 0 \mid 1 \leqq i \leqq k\right\}$ and let $H_{j}$ be the pure subgroup of $A$ generated by $S_{j}$, where $1 \leqq j \leqq 3$. We prove that $X=\left\{x_{1}, \cdots, x_{k}\right\}$ may be chosen such that $H_{j} \cap A_{k l}^{*}=0$, where $\{j, k, l\}=\{1,2,3\}$ and $A_{k l}^{*}$ is the pure subgroup of $A$ generated by $A_{k}+A_{l}$. Assume that, for example, $l_{1} x_{11}+$ $\cdots+l_{k} x_{1 k} \in A_{23}^{*}$ for $l_{i} \in Z$ and that there is a least integer $t$ with $l_{t} x_{1 t} \neq 0$. Then $\sum m_{i} l_{i} x_{i} \in A_{23}^{*}$ and $\sum r_{i} x_{i} \in A_{23}^{*}$ where, $r_{i}=\left(m_{i} l_{i}\right) / d$ and $d$ is the greatest common divisor of $\left\{m_{1} l_{1}, \cdots, m_{k} l_{k}\right\}$. But $\Sigma_{\Lambda}=\tau_{\infty}$ so there is an integer $n$ with $n\left(\sum r_{i} x_{i}\right) \in A_{2}+A_{3}$ and $n A_{1}=A_{1}$. Since $x_{i}+A_{1}+A_{2}+A_{3}$ generates $C_{i}, m_{i}$ divides $n r_{i}$ for $1 \leqq i \leqq k$. But $r_{t} \neq 0$, consequently $m_{t}$ divides $n$, and $m_{t} A_{1}=A_{1}$. Define $x_{t}^{\prime}=m_{t}^{-1}\left(x_{2 t}+x_{3 t}\right)$ a generator of $C_{t}$. Replacing $x_{t}$ by $x_{t}^{\prime}$ and repeating the argument for all nonzero $l_{i} x_{1 i}$ proves that $X$ may be chosen such that $H_{1} \cap A_{23}^{*}=0$. Since replacing $x_{t}$ by $x_{t}^{\prime}$ does not affect $S_{2}$ and $S_{3}$, similar arguments for $S_{2}$ and $S_{3}$ give the desired result. Note that, in fact, $S_{i}$ is a $Z$-independent subset of $A$ for $i=1,2,3$.

Let $G_{i}$ be the pure subgroup of $A$ generated by $\left\{x_{1 i}, x_{2 i}, x_{3 i}\right\}$. One easily proves that $G=G_{1} \oplus \cdots \oplus G_{k} \subseteq A$. Now $A_{3}=A_{3}^{\prime} \oplus\left(G \cap A_{3}\right)$ and $A_{3} \subseteq G \oplus A_{3}^{\prime} \subseteq A$. Furthermore, $A_{2}=\left(G \cap A_{2}\right) \oplus\left(A_{3}^{\prime} \cap A_{2}\right) \oplus A_{2}^{\prime}$ and $A_{2}+A_{3} \subseteq$ $G \oplus A_{3}^{\prime} \oplus A_{2}^{\prime}$. Finally, $A_{1}=\left(G \cap A_{1}\right) \oplus\left(A_{3}^{\prime} \cap A_{1}\right) \oplus\left(A_{2}^{\prime} \cap A_{1}\right) \oplus A_{1}^{\prime}$ and $A_{1}+A_{2}+$ $A_{3} \subseteq G \oplus A_{3}^{\prime} \oplus A_{2}^{\prime} \oplus A_{1}^{\prime}$. But $x_{1}, \cdots, x_{k} \in G$, so $A=G_{1} \oplus \cdots \oplus G_{k} \oplus A_{3}^{\prime} \oplus A_{2}^{\prime} \oplus A_{1}^{\prime}$. Since $A$ is indecomposable, $A=G_{k}, k=1$, and rank $A \leqq 3$.

COROLlary 1.2. Let $X_{i}$ be a rank 1 subgroup of $Q$ such that $1 \in X_{i}$ and type $\left(X_{i}\right)=\tau_{i}$ for $i=0,1,2,3$ and let $V=Q x \oplus Q y \oplus Q z$ be a $Q$-vector space of dimension 3. If $A$ is an indecomposable $\mathscr{R}_{\Lambda^{-}}$group and if $\Sigma_{\Lambda}=\tau_{\infty}$, then $A$ is isomorphic to a group in one of the following classes of subgroups of $V$ : 
(i) $X_{0} x, X_{1} x, X_{2} x, X_{3} x$;

(ii) $X_{1} x+X_{2} y+X_{0}\left((x+y) / n_{3}\right)$ where $2 \leqq n_{3}<\infty$ and $n_{3} X_{3}=X_{3}$;

(iii) $X_{1} x+X_{3} y+X_{0}\left((x+y) / n_{2}\right)$ where $2 \leqq n_{2}<\infty$ and $n_{2} X_{2}=X_{2}$;

(iv) $X_{2} x+X_{3} y+X_{0}\left((x+y) / n_{1}\right)$ where $2 \leqq n_{1}<\infty$ and $n_{1} X_{1}=X_{1}$;

(v) $X_{1} x+X_{2} y+X_{3}(x+y)$;

(vi) $X_{1} x+X_{2} y+X_{3} z+X_{0}\left((x+y+z) / n_{1} n_{2} n_{3}\right)$ where $2 \leqq n_{1} n_{2} n_{3}<\infty$ and $n_{i} X_{i}=X_{i}$.

Lemma 1.3. Let $A$ be an indecomposable $\mathscr{R}_{\Lambda}$-group with typeset $(A)=\Lambda$ and assume that if $p$ is a prime dividing the order of $A /\left(A\left(\tau_{1}\right)+A\left(\tau_{2}\right)+A\left(\tau_{3}\right)\right)$, then $p A\left(\tau_{j}\right)=A\left(\tau_{j}\right)$ for some $j$. Then $A=A_{12}^{*}+A_{13}^{*}+A_{23}^{*}$ where $A_{i j}^{*}$ is the pure subgroup of $A$ generated by $A\left(\tau_{i}\right)+A\left(\tau_{j}\right)$.

ProOF. Let $n$ be the smallest integer with $n A \subset A\left(\tau_{1}\right)+A\left(\tau_{2}\right)+A\left(\tau_{3}\right)$. The hypotheses guarantee the existence of relatively prime integers $n_{1}, n_{2}, n_{3}$ such that $n=n_{1} n_{2} n_{3}$ and $n_{j} A\left(\tau_{j}\right)=A\left(\tau_{j}\right)$.

Let $x=n^{-1}\left(a_{1}+a_{2}+a_{3}\right)$ be an arbitrary element of $A$ for some $a_{j} \in A\left(\tau_{j}\right)$. Choose integers $r_{i}$ and $s_{i}$ with $r_{1} n_{1}+s_{1} n_{2}=r_{2} n_{1}+s_{2} n_{3}=r_{3} n_{2}+s_{3} n_{3}=1$. Then $x=u+v+w$, where

$$
\begin{aligned}
u & =n_{3}^{-1}\left(n_{1}^{-1} r_{3} a_{1}+n_{2}^{-1} r_{2} a_{2}\right) \in A_{12}^{*}, \\
v & =n_{2}^{-1}\left(n_{1}^{-1} s_{3} a_{1}+n_{3}^{-1} r_{1} a_{3}\right) \in A_{13}^{*} \\
w & =n_{1}^{-1}\left(n_{2}^{-1} s_{2} a_{2}+n_{3}^{-1} s_{1} a_{3}\right) \in A_{23}^{*}
\end{aligned}
$$

Proposition 1.4. Assume that $A$ is an indecomposable $\mathscr{K}_{\Lambda}$-group with typeset $(A)=\Lambda$; that $A\left(\tau_{1}\right) \oplus A\left(\tau_{2}\right) \oplus A\left(\tau_{3}\right) \subset A$; and that if $p$ is a prime dividing the order of $\left.A /\left(A \tau_{1}\right) \oplus A\left(\tau_{2}\right) \oplus A\left(\tau_{3}\right)\right)$, then $p A\left(\tau_{j}\right)=A\left(\tau_{j}\right)$ for some $j$. Then $A \oplus A\left(\tau_{1}\right) \oplus A\left(\tau_{2}\right) \oplus A\left(\tau_{3}\right) \simeq A\left|A\left(\tau_{1}\right) \oplus A\right| A\left(\tau_{2}\right) \oplus A \mid A\left(\tau_{3}\right)$.

ProOf. Let $A_{j}=A\left(\tau_{j}\right)$. It suffices to construct a split exact sequence (*) $0 \longrightarrow A \stackrel{f_{0}}{\longrightarrow} A\left|A_{1} \oplus A / A_{2} \oplus A / A_{3} \stackrel{f_{1}}{\longrightarrow} A\right| A_{12}^{*} \oplus A / A_{13}^{*} \oplus A / A_{23}^{*} \longrightarrow 0$.

Note that $A / A_{i j}^{*}$ is quasi-isomorphic, hence isomorphic, to $A_{k}$ where $\{i, j, k\}=\{1,2,3\}$ (recall that $A_{k}$ is homogeneous completely decomposable of type $\tau_{k}$ ).

Define $f_{0}$ by letting $f_{0}(a)=\left(a+A_{1},-a+A_{2}, a+A_{3}\right)$ and define

$$
\begin{aligned}
f_{1}\left(a+A_{1}, b\right. & \left.+A_{2}, c+A_{3}\right) \\
& =\left(a+b+A_{12}^{*}, c-a+A_{13}^{*},-b-c+A_{23}^{*}\right) .
\end{aligned}
$$

Clearly $f_{0}$ and $f_{1}$ are well defined, $f_{0}$ is monic and image $f_{0} \subseteq$ kernel $f_{1}$. 
The image of $f_{0}$ is pure, for let $p$ be a prime and let $p w=f_{0}(a)$ where $w=\left(x+A_{1}, y+A_{2}, z+A_{3}\right)$. Then $p x-a=a_{1} \in A_{1}, p y+a=a_{2} \in A_{2}$, and $p z-a=a_{3} \in A_{3}$. Now $p(x+2 y+z)=a_{1}+2 a_{2}+a_{3} \in A_{1} \oplus A_{2} \oplus A_{3}$. If $x+2 y+$ $z \in A_{1} \oplus A_{2} \oplus A_{3}$, then $p^{-1} a_{j} \in A\left(\tau_{j}\right), p^{-1} a \in A$ and $w=f_{0}\left(p^{-1} a\right)$. If

$$
x+2 y+z \notin A_{1} \oplus A_{2} \oplus A_{3},
$$

then $p^{-1} a_{j} \in A\left(\tau_{j}\right)$, for some $j$ (recall hypotheses). Once again, $p^{-1} a \in A$ and $w=f_{0}\left(p^{-1} a\right)$.

The preceding remarks show that if kernel $f_{1} /$ image $f_{0}$ is torsion, then kernel $f_{1}=$ image $f_{0}$. Let $w=\left(a+A_{1}, b+A_{2}, c+A_{3}\right) \in$ kernel $f_{1}$. For some nonzero integer $m, m(a+b)=a_{1}+a_{2} \in A_{1} \oplus A_{2}, m(c-a)=b_{1}+b_{3} \in A_{1} \oplus A_{3}$ and $m(-b-c)=c_{2}+c_{3} \in A_{2} \oplus A_{3}$. But $0=m(a+b)+m(c-a)+m(-b-c)=$ $\left(a_{1}+b_{1}\right)+\left(a_{2}+c_{2}\right)+\left(b_{3}+c_{3}\right) \in A_{1} \oplus A_{2} \oplus A_{3}$, so $a_{1}=-b_{1}, \quad a_{2}=-c_{2}$ and $b_{3}=-c_{3}$. Consequently, $m a-a_{1}=-m b+a_{2}=m c-b_{3}$ and $f_{0}\left(m a-a_{1}\right)=$ $\left(m a+A_{1}, m b+A_{2}, m c+A_{3}\right)=m w$. Thus kernel $f_{1} /$ image $f_{0}$ is torsion, as desired.

To prove that $f_{1}$ is epic, let $a+A_{12}^{*} \in A \mid A_{12}^{*}$ and write $a=a_{12}^{*}+a_{13}^{*}+a_{23}^{*}$ where $a_{i j}^{*} \in A_{i j}^{*}$. Then

$$
\begin{aligned}
f_{1}\left(a_{13}^{*}+A_{1}, a_{23}^{*}+A_{2}, 0\right) & =\left(a_{13}^{*}+a_{23}^{*}+A_{12}^{*},-a_{13}^{*}+A_{13}^{*},-a_{23}^{*}+A_{23}^{*}\right) \\
& =a+A_{12}^{*} .
\end{aligned}
$$

Similarly, $A / A_{13}^{*}$ and $A / A_{23}^{*}$ are contained in the image of $f_{1}$.

Finally, we define a homomorphism $h: A\left|A_{12}^{*} \oplus A\right| A_{13}^{*} \oplus A\left|A_{23}^{*} \rightarrow A\right| A_{1} \oplus$ $A\left|A_{2} \oplus A\right| A_{3}$. Note that $f_{1}: A_{13}^{*} / A_{1} \oplus A_{23}^{*} / A_{2} \rightarrow A \mid A_{12}^{*}$ is an epimorphism between two homogeneous completely decomposable groups of type $\tau_{3}$. Thus there is a homomorphism $h_{3}: A \mid A_{12}^{*} \rightarrow A_{13}^{*} / A_{1} \oplus A_{23}^{*} / A_{2}$ with $f_{1} h_{3}=1$ (see Fuchs [4, p. 163] or [1]). Similarly there are maps $h_{2}: A \mid A_{13}^{*} \rightarrow A_{12}^{*} / A_{1} \oplus$ $A_{23}^{*} / A_{3}$ and $h_{1}: A \mid A_{23}^{*} \rightarrow A_{13}^{*} / A_{3} \oplus A_{12}^{*} / A_{2}$ with $f_{1} h_{2}=1$ and $f_{1} h_{1}=1$. Define $h=h_{3}+h_{2}+h_{1}$, and observe that $f_{1} h=1$. Thus $(*)$ is a split exact sequence.

ProOF OF THEOREM 2(b). A consequence of the fact that $\Sigma_{\Lambda}=\tau_{\infty}$ and Proposition 1.4.

Proof of Corollary 3. (a) If $A$ is a $\mathscr{C}_{3}$-group, then typeset $(A) \subseteq$ $\left\{\tau_{p} \cap \tau_{q} \cap \tau_{r}, \tau_{p} \cap \tau_{q}, \tau_{q} \cap \tau_{r}, \tau_{p} \cap \tau_{r}, \tau_{p}, \tau_{q}, \tau_{r}\right\}$. Suppose, for example, that $\tau_{p} \in \operatorname{typeset}(A)$. Let $R$ be a rank 1 subring of $Q$ such that $R$ has type $\tau_{p}$ (e.g., $R=Z_{p}$, the localization of $Z$ at $p$ ). Then $A\left(\tau_{p}\right)$ is a free $R$-module, $R \otimes_{Z} A\left(\tau_{p}\right) \simeq A\left(\tau_{p}\right)$ and $R \otimes_{Z} A=F \oplus D$, where $F$ is a free $R$-module and $D$ is divisible. It follows that $R \otimes_{Z} A\left(\tau_{p}\right)$ is a summand of $R \otimes_{Z} A$. Under the canonical imbedding, $R \otimes_{Z} A\left(\tau_{p}\right)=A\left(\tau_{p}\right) \subset A \subset R \otimes_{Z} A$ so that $A\left(\tau_{p}\right)$ is a summand of $A$. Thus $A=A\left(\tau_{p}\right) \oplus A\left(\tau_{q}\right) \oplus A\left(\tau_{r}\right) \oplus G$ and $\tau_{p}, \tau_{q}, \tau_{r} \notin$ typeset $G$. 
(b) Let $\tau_{1}=\tau_{p} \cap \tau_{q}, \tau_{2}=\tau_{p} \cap \tau_{r}, \tau_{3}=\tau_{q} \cap \tau_{r}$. If $B$ is an indecomposable rank $2 \mathscr{C}_{3}$-group, then $B$ is in class (see Corollary 1.2)

(ii) iff $B /\left(B\left(\tau_{1}\right) \oplus B\left(\tau_{2}\right)\right) \simeq Z / p^{\alpha} Z, 1 \leqq \alpha<\infty$;

(iii) iff $B /\left(B\left(\tau_{1}\right) \oplus B\left(\tau_{3}\right)\right) \simeq Z / q^{\beta} Z, 1 \leqq \beta<\infty$;

(iv) iff $B /\left(B\left(\tau_{2}\right) \oplus B\left(\tau_{3}\right)\right) \simeq Z / r^{\gamma} Z, 1 \leqq \gamma<\infty$; and

(v) iff $B /\left(B\left(\tau_{1}\right) \oplus B\left(\tau_{2}\right)\right) \simeq Z\left(p^{\infty}\right)$ (the $p$-divisible subgroup of $Q / Z$ ).

Let $A=G_{1} \oplus \cdots \oplus G_{k}=H_{1} \oplus \cdots \oplus H_{l}$ be a decomposition of $A$ into indecomposable summands, where rank $G_{i} \leqq 2$ and rank $H_{i} \leqq 2$. Now $A /\left(A\left(\tau_{1}\right)+A\left(\tau_{2}\right)\right)$ is a direct sum of a finite number of cyclic $p$-groups, a finite number of rank 1 groups of types $\tau_{p}, \tau_{q}, \tau_{r}, \tau_{0}$ and $\tau_{3}$ and a finite number of copies of $Z\left(p^{\infty}\right)$. Moreover, $A /\left(A\left(\tau_{1}\right)+A\left(\tau_{2}\right)+A\left(\tau_{3}\right)\right)$ is the direct sum of a finite number of cyclic $p$-groups, a finite number of cyclic $q$-groups, a finite number of cyclic $r$-groups, and a finite number of rank 1 groups of type $\tau_{p}, \tau_{q}, \tau_{r}$ and $\tau_{0}$. Thus $k=l$ and $G_{i} \simeq H_{\sigma(i)}$ for some permutation $\sigma$ of $\{1,2, \cdots, k\}$.

(c) A consequence of Theorem 2(b) and Corollary 3(b).

2. Examples. Let $\Lambda=\left\{\tau_{0}, \tau_{1}, \tau_{2}, \tau_{3}\right\}$ where $\tau_{0}=\tau_{1} \cap \tau_{2}=\tau_{1} \cap \tau_{3}=\tau_{2} \cap$ $\tau_{3}$; let $\lambda=\left\{\tau_{0}, \tau_{1}, \tau_{2}\right\}$; let $X_{i}$ be a rank 1 subgroup of $Q$ with $1 \in X_{i}$ and with type $\left(X_{i}\right)=\tau_{i}$; and let $V=Q w \oplus Q x \oplus Q y \oplus Q z$.

EXAMPLE 2.1. If $\sum_{\lambda} \neq \infty$, then there is a rank 2 indecomposable $\mathscr{R}$ group $A$ with typeset $(A)=\lambda$.

Proof. Choose a prime $p$ with $p X_{i} \neq X_{i}$, where $i=1,2$, and let $A=$ $X_{1} w+X_{2} x+X_{0}((w+x) / p) \subset V$.

EXAMPLE 2.2. If $\sum_{\lambda} \neq \tau_{\infty}$ and $\sum_{\lambda} \neq \tau_{p}$ for all primes $p$, then there is an $\mathscr{R}$-group $A$ with typeset $(A)=\lambda$ such that $A=A_{1} \oplus A_{2}=A_{3} \oplus B_{1} \oplus B_{2}$, where each $A_{i}$ is an indecomposable group of rank 2 and $B_{1} \oplus B_{2}$ is completely decomposable.

Proof. Choose distinct primes $p$ and $q$ with $p X_{i} \neq X_{i}$ and $q X_{i} \neq X_{i}$ for $i=1$, 2. Define $A_{1}=X_{1} w+X_{2} x+X_{0}((w+x) / p), A_{2}=X_{1} y+X_{2} z+X_{0}((y+z) / q)$ and let $A=A_{1} \oplus A_{2}$. Let $C_{1}$ and $C_{2}$ be the pure subgroups of $A$ generated by $q w+p y$ and $q x+p z$, respectively. Define $A_{3}$ to be the pure subgroup of $A$ generated by $C_{1} \oplus C_{2}$ and note that $A_{3}\left(\tau_{1}\right)=C_{1} \oplus B_{1}$ and $A_{3}\left(\tau_{2}\right)=C_{2} \oplus B_{2}$, for some $B_{j} \subset A\left(\tau_{j}\right)$. Clearly $A_{3} \cap\left(B_{1} \oplus B_{2}\right)=0$ so that $A\left(\tau_{1}\right) \oplus A\left(\tau_{2}\right) \subseteq$ $A_{3} \oplus B_{1} \oplus B_{2} \subseteq A$. But

$$
a=p^{-1}(w+x)+q^{-1}(y+z)=((q w+p y)+(q x+p z)) p^{-1} q^{-1} \in A_{3}
$$

and the order of $a+A\left(\tau_{1}\right)+A\left(\tau_{2}\right)$ is $p q$ in $A /\left(A\left(\tau_{1}\right) \oplus A\left(\tau_{2}\right)\right)$ so that $A=A_{1} \oplus A_{2}=A_{3} \oplus B_{1} \oplus B_{2}$.

EXAMPLE 2.3. If $\sum_{\Lambda} \neq \tau_{\infty}$, then there is an indecomposable rank 3 $\mathscr{R}$-group $A$ such that typeset $(A)=\Lambda$ and $A \oplus H$ is not a direct sum of 
groups of rank $\leqq 2$ for all finite rank completely decomposable $\mathscr{R}_{\Lambda^{-}}$ groups $H$.

Proof. Choose a prime $p$ with $p X_{i} \neq X_{i}$ for $i=1,2,3$ and let $A=$ $X_{1} w+X_{2} x+X_{3} y+X_{0}((w+x+y) / p)$. Note that $A$ is indecomposable and $(w+x+y) / p \notin A_{12}^{*}+A_{13}^{*}+A_{23}^{*}$. If $A \oplus H=G_{1} \oplus \cdots \oplus G_{k}$, where $H$ is a finite rank completely decomposable $\mathscr{R}_{\Lambda}$-group and rank $G_{i} \leqq 2$, then one can easily prove that $A=A_{12}^{*}+A_{13}^{*}+A_{23}^{*}$, a contradiction.

EXAMPLE 2.4. If $\sum_{\Lambda} \neq \tau_{\infty}$, then there is an indecomposable rank $3 \mathscr{R}$ group $A$ such that typeset $(A)=\Lambda$ and $A$ is not quasi-isomorphic to a completely decomposable group (see Corollary 1.2).

PROOF. Let $A^{\prime}=X_{1} w+X_{2} x+X_{3}(w+x)$, a strongly indecomposable $\mathscr{R}_{\Lambda}$-group of rank 2. Let $A=A^{\prime}+X_{3} y+X_{0}((w+x+y) / p)$ where $p$ is a prime with $p X_{i} \neq X_{i}$ for $i=1,2,3$. Then $A$ is indecomposable of rank 3, $A$ is quasi-isomorphic to $A^{\prime} \oplus X_{3}$, and $A^{\prime}$ is not quasi-isomorphic to a completely decomposable group.

\section{REFERENCES}

1. D. Arnold and E. L. Lady, Endomorphism rings and direct sums of torsion free Abelian groups (to appear).

2. M. C. R. Butler, A class of torsion-free Abelian groups of finite rank, Proc. London Math. Soc. (3) 15 (1965), 680-698. MR 36 \#1532.

3. T. B. Cruddis, On a class of torsion-free Abelian groups, Proc. London Math. Soc. (3) 21 (1970), 243-276. MR 42 \#6104.

4. L. Fuchs, Abelian groups, Akad. Kiado, Budapest, 1958; republished by Internat. Series of Monos on Pure and Appl. Math., Pergamon Press, New York, 1960. MR 21 \#5672; 22 \#2644.

5. - Infinite Abelian groups. Vol. I, Pure and Appl. Math., vol. 36, Academic Press, New York, 1970. MR 41 \#333.

Department of Mathematical Sciences, New Mexico State University, Las Cruces, New Mexico 88001 